\title{
A comparison study of SARS-CoV-2 IgG antibody between male and female COVID-19 patients: A possible reason underlying different outcome between sex
}

\author{
Fanfan Zeng ${ }^{1}$ (1) | Chan Dai ${ }^{2}$ | Pengcheng Cai ${ }^{1}$ | Jinbiao Wang ${ }^{1}$ | Lei $\mathrm{Xu}^{1}$ | \\ Jianyu $\mathrm{Li}^{1}$ | Guoyun $\mathrm{Hu}^{1}$ | Zheng Wang ${ }^{3}$ | Fang Zheng ${ }^{2}$ | Lin Wang ${ }^{1}$
}

${ }^{1}$ Department of Clinical Laboratory, Union Hospital, Tongji Medical College, Huazhong University of Science and Technology, Wuhan, Hubei, China

${ }^{2}$ Department of Immunology, School of Basic Medicine, Tongji Medical College, Huazhong University of Science and Technology, Wuhan, Hubei, China

${ }^{3}$ Department of Gastrointestinal Surgery, Union Hospital, Tongji Medical College, Huazhong University of Science and

Technology, Wuhan, Hubei, China

\section{Correspondence}

Zheng Wang, Department of Gastrointestinal Surgery, Union Hospital, Tongji Medical College, Huazhong University of Science and Technology, Jiefang Avenue 1277, Wuhan 430022, China. Email: zhengwang@hust.edu.cn

Fang Zheng, Department of Immunology, School of Basic Medicine, Tongji Medical College, Huazhong University of Science and Technology, Hangkong Road 13, Wuhan 430030, China.

Email: zhengfangtj@hust.edu.cn

Lin Wang, Department of Clinical Laboratory, Union Hospital, Tongji Medical College, Huazhong University of Science and Technology, Jiefang Avenue 1277, Wuhan 430022, China.

Email: lin_wang@hust.edu.cn

\begin{abstract}
The emergence of severe acute respiratory syndrome coronavirus 2 (SARS-CoV-2) in China at the end of 2019 has spread throughout the world and caused many thousands of deaths. The previous study reported a higher severe status rate and mortality rate in male patients in China. However, the reason underlying this difference has not been reported. The convalescent plasma containing a high level of SARS-CoV-2 immunoglobulin G (IgG) antibody has been used in clinical therapy and achieved good effects in China. In this study, to compare the differences of the SARS-CoV-2 IgG antibody between male and female patients, a total number of 331 patients confirmed SARS-CoV-2 infection were enrolled. The serum of these patients was collected during hospitalization and detected for the SARS-CoV-2 IgG antibody. Our data showed that the concentration of IgG antibody in mild, general, and recovering patients showed no difference between male and female patients. In severe status, compared with male patients, there were more female patients having a relatively high concentration of serum SARS-CoV-2 IgG antibody. In addition, the generation of IgG antibody in female patients was stronger than male patients in disease early phase. Our study identified a discrepancy in the SARS-CoV-2 IgG antibody level in male and female patients, which may be a potential cause leading to a different outcome of Coronavirus Disease 2019 between sex.
\end{abstract}

KEYWORDS

COVID-19 patients, SARS-CoV-2 IgG antibody, sexual discrepancy

\section{1 | INTRODUCTION}

In December 2019, an acute respiratory disease caused by an unknown respiratory syndrome coronavirus (severe acute respiratory syndrome coronavirus [SARS-CoV])-2 emerged in Wuhan, China. ${ }^{1}$ The pneumonia was then officially named as Coronavirus Disease 2019 (COVID-19) by the World Health Organization. The incubation time of COVID-19 is generally 3 to 7 days, and the typical clinical manifestations are fever, cough, shortness of breath, and fatigue., ${ }^{2,3}$ By 22 April 2020, more than 84000 confirmed cases and 4600 dead cases have been reported in China. ${ }^{4}$ About $80 \%$ of the patients developed mild or general symptoms and $20 \%$ more or less patients progressed to severe or critical disease. ${ }^{2}$ The previous study reported that male patients were susceptible to develop a more severe symptom and had a higher mortality rate compared with female patients in China. ${ }^{2,4,5}$ However, the causes underlying such a difference have not been reported. 
The detection of serum SARS-CoV-2 specific antibody has been added to the New Coronavirus Pneumonia Prevention and Control Program (7th edition) and is now widely used in China for clinical diagnosis of SARS-CoV-2 infection. ${ }^{6}$ The SARS-CoV-2 specific immunoglobulin $M$ and immunoglobulin $G$ (IgG) antibodies were observed in about 1 week after symptom onset. ${ }^{7}$ Due to the protective role of the SARS-CoV-2 IgG antibody and currently no effective treatment for the patients in severe and critical status, the plasma donated by cured patients which contain high levels of SARS-CoV-2 IgG antibody is now used for transferring therapy in China. ${ }^{8-10}$ As a consequence, the treated patients showed a significant elimination of virus and an obvious attenuation in disease symptoms. ${ }^{10}$ However, whether the level of the SARS-CoV-2 IgG antibody exists a difference between male and female patients remains unclear.

In this study, by detecting and analyzing the SARS-CoV-2 IgG antibody in recovering, mild, general, and severe status patients, we identified that there were more female patients generating a high level of IgG antibody relative to male patients in severe status. In addition, the production of IgG antibody tended to be stronger in female patients in the early phase of COVID-19. The higher antibody concentration in female cases may play an important role in preventing patients from progressing to a severe status and even death. Our study suggests that the discrepancies of the SARS-CoV-2 IgG antibody generated in male and female patients may be a potential cause resulting in the different outcomes of COVID-19 between sex.

\section{2 | METHODS}

\section{1 | Patients}

All the cases used in this study were derived from Wuhan Union Hospital, Tongji Medical College of Huazhong University of Science and Technology. The general information, clinical diagnosis, and pathogenic diagnosis of patients were extracted from the electronic medical records system. All of the enrolled patients were confirmed cases and classified by the New Coronavirus Pneumonia Prevention and Control Program (7th edition). This study was approved by the Ethics Committee of Wuhan Union Hospital, Tongji Medical College, Huazhong University of Science and Technology and performed in accordance with the tenets of the Declaration of Helsinki. The patients enrolled in the study signed informed consent forms.

\section{2 | Anti-SARS-CoV-2 IgG antibody detection}

Blood samples were centrifuged at room temperature and the supernatant serum was used for detection. Serum anti-SARS-CoV-2 IgG was measured by chemiluminescent microparticle immunoassay using the commercially available kit on iFlash 3000 chemiluminescent immune analyzer (YHLO Biotechnology Co., Ltd., Shenzhen, China). The cat number of the kit is C86095G. Briefly, serum and SARS-CoV-2 antigen-coated paramagnetic particles were incubated for 20 minutes. After washing, the mouse anti-human IgG antibody labeled with acridinium was added and incubated for 4 minutes. Following another washing cycle, pretrigger and trigger solutions were added to the reaction mixture. The resulting chemiluminescent reaction was measured as relative luminescence intensity by the iFlash 3000 chemiluminescent immune analyzer. All operations in the detection were in strict accordance with the manufacturer's protocols.

\section{3 | Statistics}

In this study, GraphPad 6.0 was applied for mapping and data statistical analysis. The Mann-Whitney $U$ test was used to compare SARS-CoV-2 IgG antibody concentration between two groups. $A P$ value less than .05 was considered statistically significant.

\section{3 | RESULTS}

In this study, a total number of 331 in hospital patients were enrolled, among which male and female patients were 127 and 204, respectively. These COVID-19 patients contained three disease severity status (mild, general, and severe) and recovering patients. The number of these four were, respectively, 22, 87, 22, and 200. In mild group, male patients accounted for $36.4 \%$ and female patients accounted for $63.6 \%$. The average age of male (M) and female (F) patients were 45.2 and 42.2 years old. In general group, male patients and female patients took up for $42.5 \%$ and $57.5 \%$, respectively. The mean age of male and female patients were 46.2 and 49.4. In severe patients, the enrolled male and female cases were equal and the average age were 59.4 and 63.1. The recovering patients were about to be discharged from the hospital and were once diagnosed as a mild or general symptom. For recovering cases, male and female patients occupied respectively $35.5 \%$ and $64.5 \%$, and the average age were nearly the same. As the same with the previous study reported, elderly people enrolled in our study were more susceptible to developing severe status.

To clarify the dynamic change of the SARS-CoV-2 IgG antibody in male and female patients, we analyzed the concentration of the SARS-CoV-2 IgG antibody between male and female patients in those four disease status. As shown in Figure 1A, In female patients, the concentration of SARS-CoV-2 IgG antibody continuously increased from mild status patients to general and severe status patients, and then decreased in recovering patients. While in male patients, the lgG antibody raised from mild status patients to general status patients, and then decreased from general status patients to recovering patients. In addition to severe status patients, the concentration of the SARS-CoV-2 IgG antibody in the other three statuses were nearly the same. While in severe status, compared with male patients, the concentration of the SARS-CoV-2 IgG antibody in severe status was higher in female patients. Next, we mapped scatter diagrams respectively for the above four status of patients. 
(A)

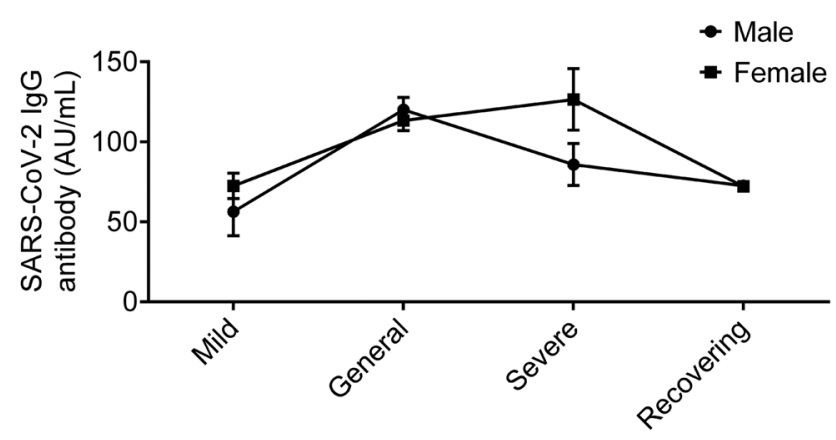

(C)

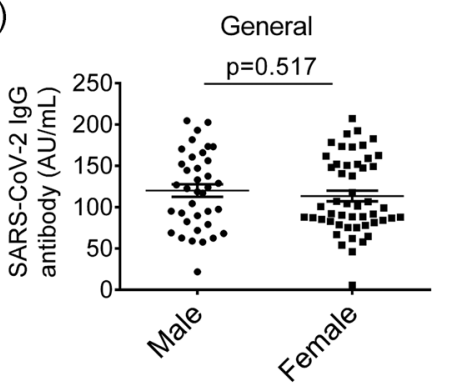

(F)

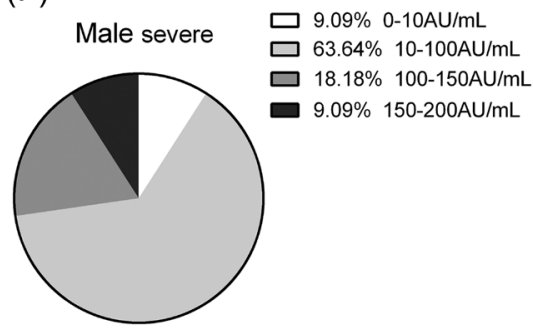

(D)

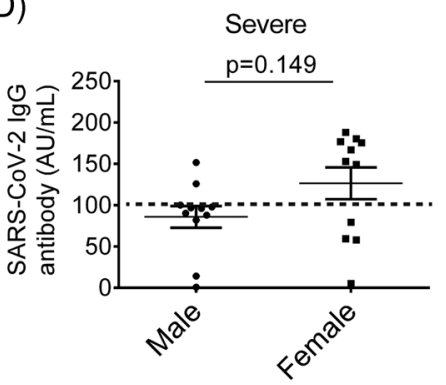

(B)

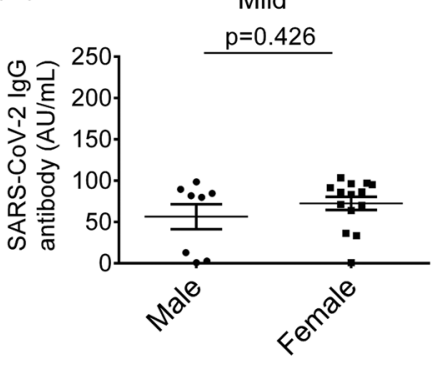

$(\mathrm{E})$

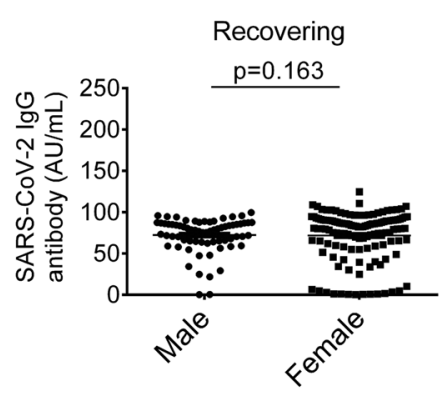

(G)

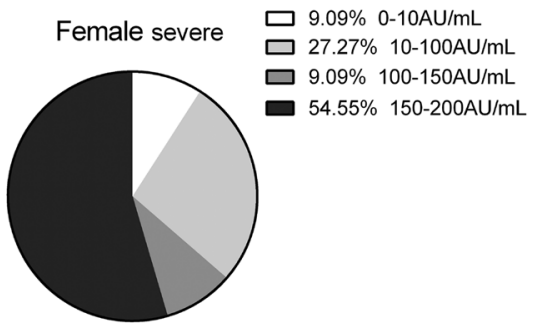

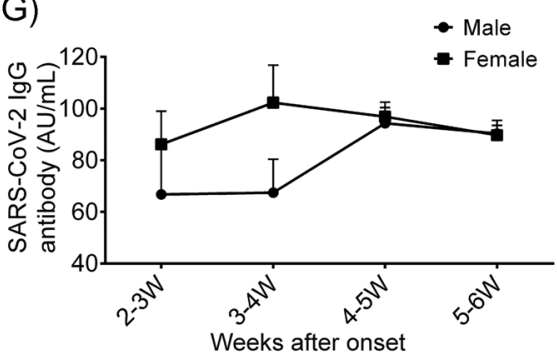

FIGURE 1 SARS-CoV-2 IgG antibody showed enrichment in high levels in severe status and strong production in the early phase in female patients. A, Average concentration of SARS-CoV-2 IgG antibody in four status of COVID-19 in male and female patients. B-E, Comparison of SARS-CoV-2 IgG antibody concentration between male and female patients in mild (B), general (C), severe (D), and recovering status patients (E). F, percentage analysis of different concentration rage of IgG antibody in male (left) and female (right) patients in severe status. (G) Average concentration of the SARS-CoV-2 IgG antibody in indicate time points after disease onset. Patients were divided into four groups according to the time after disease onset. The IgG level was analyzed, respectively, in male and female patients. $n(2-3 \mathrm{~W}, 3-4 \mathrm{~W}, 4-5 \mathrm{~W}, 5-6 \mathrm{~W})=8,10,49,42$ for male and 11, 15, 48, 76 for female. All data are expressed as mean \pm SEM. IgG, immunoglobulin G; SARS-CoV-2, severe acute respiratory syndrome coronavirus 2; SEM, standard error of the mean

However, none of these four status showed a statistical difference in SARS-CoV-2 IgG antibody concentration between male and female patients (Figure 1B-E). Notably, in severe status, the SARS-CoV-2 IgG antibody in most female patients was more than $100 \mathrm{AU} / \mathrm{mL}$, while in male patients, most of the IgG antibody were under $100 \mathrm{AU} / \mathrm{mL}$ (Figure 1D). Next, to determine the concentration distribution of the SARS-CoV-2 IgG antibody, we analyzed the percentage of IgG antibody in each concentration range $(0-10 \mathrm{AU} / \mathrm{mL}, 10-100 \mathrm{AU} / \mathrm{mL}$, 100-150 AU/mL, and 150-200 AU/mL) in male and female patients. As shown in Figure $1 \mathrm{~F}$, The percentage of range 0 to $10 \mathrm{AU} / \mathrm{mL}$ was equal in male and female patients and the percentage of range 100 to $150 \mathrm{AU} / \mathrm{mL}$ was also close between male and female patients. While the percentage of range 10 to $100 \mathrm{AU} / \mathrm{mL}$ and 150 to $200 \mathrm{AU} / \mathrm{mL}$ showed a great difference between the two groups. In range 10 to $100 \mathrm{AU} / \mathrm{mL}$, the male patients accounted for as much as $63.64 \%$, while the female patient took up $27.27 \%$. In range 150 to $200 \mathrm{AU} / \mathrm{mL}$, the male patients occupied only $9.09 \%$, while the female patients accounted for as much as $54.55 \%$. These data suggest that there were more female patients generating a high level of SARS-CoV-2 IgG antibody relative to male patients in severe status. In addition, to analysis the antibody response in male and female patients, we collected the time information of each case and analyzed the concentration of SARS-CoV-2 IgG after disease onset. The result showed that the concentration of the SARS-CoV-2 IgG antibody in female patients tended to be higher than male patients in 2 to 4 weeks after disease onset, and the difference in antibody concentration disappeared after 4 weeks of disease onset (Figure 1G). Together, the above data indicated that the generation of the SARS-CoV-2 IgG antibody differs between male and female patients. Compared with male patients, there were more female patients in severe cases generating a relatively high level of SARS-CoV-2 IgG antibody and the IgG antibody presented a stronger production in female patients in disease early phase. 
TABLE 1 Basic information of patients enrolled

\begin{tabular}{llll|}
$\begin{array}{l}\text { Disease severity } \\
\text { status }\end{array}$ & $\begin{array}{l}\text { Male } \\
(n=127)\end{array}$ & $\begin{array}{l}\text { Female } \\
(n=204)\end{array}$ & $\begin{array}{l}\text { Average age } \\
(M / F)\end{array}$ \\
\hline Mild $(n=22)$ & $8(36.4 \%)$ & $14(63.6 \%)$ & $45.2 / 42.2$ \\
\hline General $(n=87)$ & $37(42.5 \%)$ & $50(57.5 \%)$ & $46.2 / 49.4$ \\
\hline Severe $(n=22)$ & $11(50 \%)$ & $11(50 \%)$ & $59.4 / 63.1$ \\
\hline Recovering $(n=200)$ & $71(35.5 \%)$ & $129(64.5 \%)$ & $49.6 / 50.1$ \\
\hline
\end{tabular}

Note: Data are expressed as mean and percentage. M/F means male/female.

\section{4 | DISCUSSION}

Currently, the COVID-19 has widespread many countries in the world, caused more than 2700000 infections and 190000 deaths. According to the latest data from the National Health Commission of China, the case fatality ratio of COVID-19 in China was about $5.6 \%$. Among the diagnosed patients, about $80 \%$ were just showed mild to moderate symptoms and had a good prognosis. ${ }^{11}$ However, for patients who progressed to severe or critical status, the mortality rates significantly increased to as high as $49 \% .^{5}$ So far there was no effective treatment for COVID-19 patients, especially for severe and critical patients. ${ }^{12}$ So it is urgent to distinguish and intervene in advance to prevent those patients who were likely to progress to severe and critical status.

The previous study reported a high severe rate and death rate in male patients relative to female patients. ${ }^{2,4,5}$ However, the possible causes underlying has not been reported. Here we identified that the production and dynamics of the SARS-CoV-2 IgG antibody were different between male and female patients. In the severe status, compared with male patients, more female patients generated a highlevel SARS-CoV-2 IgG antibody. In the 2 to 4 weeks after disease onset, the IgG antibody presented a stronger production in female patients. A recent study published in The Lancet journal showed that the first 2 to 3 weeks were a key period for those survivors. ${ }^{5}$ Thus the higher level of SARS-CoV-2 IgG antibody in a female patient in disease early phase may play an important role in reducing critical case and mortality rate. Just as a recent study reported, the lack of the SARS-CoV-2 antibody is an important factor leading to disease aggravation. ${ }^{13}$ The convalescent plasma containing a high level of this specific IgG antibody was recently used in severe and critical patients in China and was proved to be a promising therapy for COVID-19. ${ }^{10,14}$ Considering the protective role of SARS-CoV-2 IgG antibody, we believe that the different dynamics of SARS-CoV-2 IgG antibody between male and female patients may be good reasons to explain the different outcome between male and female COVID-19 patients. However, the exact mechanism contributing to this different outcome remains elusive and needs further investigation. Our study proposes that convalescent plasma should be used in advance especially for patients whose SARS-CoV-2 specific lgG antibodies are at low levels to prevent the disease from progressing to severe and critical status if the convalescent plasma is available. In addition, monitoring the SARSCoV-2 antibody for routine examination may be an efficient way to predict the progression of COVID-19 patients (Table 1).

It should be noted that there were some limitations in this study. First, as all the cases were collected in a signal hospital (Wuhan Union Hospital), the sample size was relatively small. Second, probably due to the limited sample size, there were no critical cases enrolled in this study. A comprehensive study on larger cases of male and female patients containing each status would help to understand the causes underlying the different outcomes between sex as well as the role of the SARS-CoV-2 IgG antibody in COVID-19.

In conclusion, in this study, we detected and analyzed the SARSCoV-2 IgG antibody of 331 patients and found that, compared with male patients, more female patients generated a relatively high level of IgG antibody after SARS-CoV-2 infection in severe status, and the production of IgG in female patients was stronger than male patients in the early phase of COVID-19. We propose that more attention should be paid to the patients whose IgG antibody was at low levels and monitoring the $\lg G$ antibody may be a potential method to predict COVID-19 prognosis.

\section{ACKNOWLEDGMENTS}

We thank all of the doctors, nurses, and public health workers for their efforts in fighting against SARS-CoV-2 and saving the lives of COVID-19 patients. The project was supported by the Department of Clinical Laboratory, Union Hospital, Tongji Medical College, Huazhong University of Science and Technology.

\section{CONFLICT OF INTERESTS}

The authors declare that there are no conflict of interests.

\section{AUTHOR CONTRIBUTIONS}

FZ, PC, JW, LX, JL, and GH contributed to the collection and detection of clinical samples. $F Z$ and $C D$ analyzed the data and wrote the manuscript. ZW, FZ, and LW conceived and supervised the project.

\section{ORCID}

Fanfan Zeng (D) http://orcid.org/0000-0002-4731-7641

\section{REFERENCES}

1. Chan JFW, Yuan S, Kok KH, et al. A familial cluster of pneumonia associated with the 2019 novel coronavirus indicating person-toperson transmission: a study of a family cluster. Lancet. 2020; 395(10223):514-523.

2. Huang C, Wang Y, Li X, et al. Clinical features of patients infected with 2019 novel coronavirus in Wuhan, China. Lancet. 2020;395(10223): 497-506.

3. Wu F, Zhao S, Yu B, et al. A new coronavirus associated with human respiratory disease in China. Nature. 2020;579(7798):265-269.

4. Guan W, Ni Z, Hu Y, et al. Clinical characteristics of Coronavirus Disease 2019 in China. N Engl J Med. 2020;382:1708-1720.

5. Zhou F, Yu T, Du R, et al. Clinical course and risk factors for mortality of adult inpatients with COVID-19 in Wuhan, China: a retrospective cohort study. The Lancet. 2020;395(10229):P1054-1062. 
6. Zeng $\mathrm{H}, \mathrm{Xu} \mathrm{C}$, Fan J, et al. Antibodies in infants born to mothers with COVID-19 pneumonia. JAMA. 2020;323(18):1848-1849.

7. Xiang $F$, Wang $X$, He $X$, et al. Antibody detection and dynamic characteristics in patients with COVID-19. Clin Infect Dis. 2020.

8. Shen C, Wang Z, Zhao F, et al. Treatment of 5 critically III patients with COVID-19 with convalescent plasma. JAMA. 2020;323:1582.

9. Li H, Wang YM, Xu JY, Cao B. [Potential antiviral therapeutics for 2019 Novel Coronavirus]. Zhonghua Jie He He Hu Xi Za Zhi. 2020; 43(3):170-172.

10. Ye M, Fu D, Ren Y, et al. Treatment with convalescent plasma for COVID-19 patients in Wuhan, China. J Med Virol. 2020.

11. Novel Coronavirus Pneumonia Emergency Response Epidemiology Team. [The epidemiological characteristics of an outbreak of 2019 novel coronavirus diseases (COVID-19) in China]. Zhonghua Liu Xing Bing Xue Za Zhi. 2020;41(2):145-151.

12. Dong L, Hu S, Gao J. Discovering drugs to treat coronavirus disease 2019 (COVID-19). Drug Discov Ther. 2020;14(1):58-60.
13. Cassaniti I, Novazzi F, Giardina F, et al. Performance of VivaDiag COVID-19 IgM/lgG Rapid Test is inadequate for diagnosis of COVID-19 in acute patients referring to emergency room department. J Med Virol. 2020.

14. Chen L, Xiong J, Bao L, Shi Y. Convalescent plasma as a potential therapy for COVID-19. Lancet Infect Dis. 2020;20:398-400.

How to cite this article: Zeng F, Dai C, Cai P, et al. A comparison study of SARS-CoV-2 IgG antibody between male and female COVID-19 patients: A possible reason underlying different outcome between sex. J Med Virol. 2020;92:

2050-2054. https://doi.org/10.1002/jmv.25989 